\title{
Detecção de ambliopia, ametropias e fatores ambliogênicos em comunidade assistida por Programa da Saúde da Família no Rio de Janeiro, Brasil
}

\author{
Amblyopia, ametropia and amblyogenic factors \\ detection in a community assisted by \\ Health Family Program in Rio de Janeiro, Brazil
}

Arlette Machado Oliveira1 ${ }^{1}$ Bruno Moura Fernandes ${ }^{2}$, Lucas Costa ${ }^{3}$, Andréia Lima ${ }^{4}$, Abelardo de Souza Couto Junior ${ }^{5}$, Arlindo Portes ${ }^{6}$

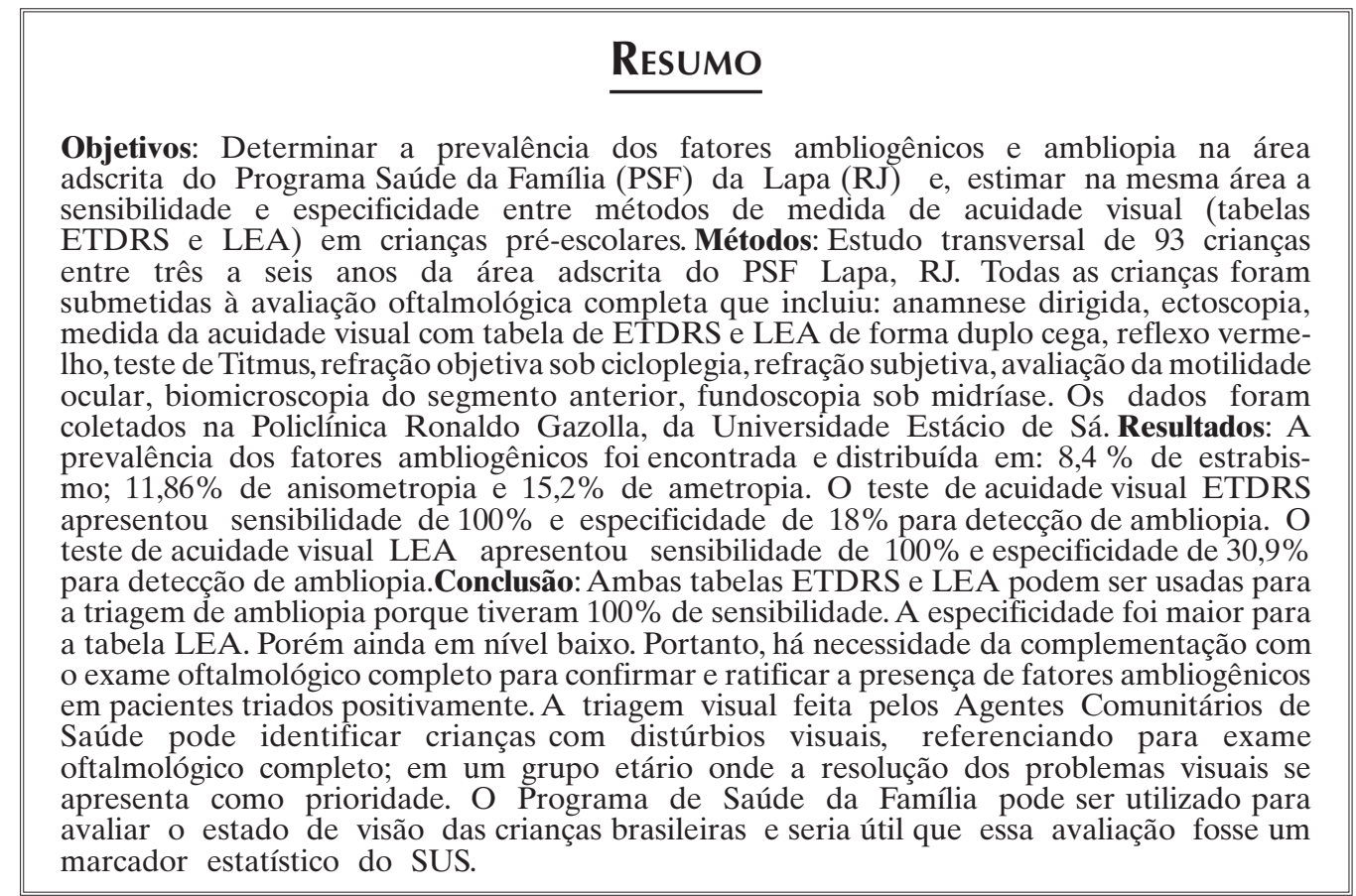

Descritores: Ambliopia/diagnóstico; Prevalência; Acuidade visual; Programa saúde da família; Criança; Pré-escolar; Análise transversal; Sensibilidade e especificidade

\footnotetext{
${ }^{1}$ Mestre em Saúde da Família, Médica oftalmologista e preceptora da Universidade Estácio de Sá - UNESA - Rio de Janeiro (RJ), Brasil; ${ }^{2}$ Acadêmico do oitavo período do curso de Medicina da Universidade Estácio de Sá - UNESA - Rio de Janeiro (RJ), Brasil; ${ }^{3}$ Acadêmico do oitavo período do curso de Medicina da Universidade Estácio de Sá - UNESA - Rio de Janeiro (RJ), Brasil; ${ }^{4}$ Acadêmica do nono período do curso de Medicina da Universidade Estácio de Sá - UNESA - Rio de Janeiro (RJ), Brasil; ${ }^{5}$ Doutor, Professor Adjunto da Faculdade de Medicina de Valença - Rio de Janeiro (RJ), Brasil;

${ }^{6}$ Doutor, Professor Adjunto da Faculdade de Medicina da Universidade Estácio de Sá - UNESA - Rio de Janeiro (RJ), Brasil.

Trabalho realizado na Universidade Estácio de Sá - UNESA - Rio de Janeiro (RJ), Brasil.

Os autores declaram inexistir conflitos de interesse.
}

Recebido para publicação em: 8/2/2010 - Aceito para publicação em 10/3/2010 


\section{INTRODUÇÃO}

$\mathbf{N}$ a idade escolar, cerca de $20-25 \%$ de crianças apresentam algum tipo de problema ocular, sendo dignos de nota os vícios de refração (miopia, hipermetropia, astigmatismo), a ambliopia e o estrabismo $^{(1)}$.

A ambliopia é a deficiência do desenvolvimento normal do sistema visual durante o período de maturação do sistema nervoso central (especificamente para o sistema visual) que se estende até os seis ou sete anos de idade, sem que haja lesão orgânica ou com uma lesão orgânica desproporcional à intensidade da baixa visual ${ }^{(2)}$.

A ambliopia é a maior responsável por visão reduzida unilateralmente durante a fase pré-escolar do que outras causas combinadas, com a prevalência de $2 \mathrm{a}$ $4 \%$ na América do Norte ${ }^{(3)}$.

São três as origens da ambliopia: privação de luz, privação de forma e perda da interação binocular.

A anisometropia pode causar ambliopia em olhos em que não há deprivação de luz, mas de forma. A privação de forma é um fator de ruptura da interação binocular provocando o aparecimento de supressão, dominância e perda de função de células corticais responsáveis pela acuidade visual central ${ }^{(4)}$.

O estudo oftalmológico de Romani em Santa Catarina, no ano de 1981, demonstrou em sua amostra que $80,5 \%$ das crianças examinadas poderiam ter suas deficiências escolares explicadas pela baixa acuidade visual ou por vícios de refração ${ }^{(5)}$. Os erros refrativos são a causa mais comum de redução visual em crianças. Entretanto, a criança geralmente não relata aos professores ou familiares os seus problemas visuais ${ }^{(6)}$.

No estrabismo unilateral o que determina a ambliopia é a perda da interação binocular. Como mecanismo de adaptação sensorial ao estado do desvio oculomotor ocorre a supressão ${ }^{(7)}$.

No estrabismo alternante ocorre correspondência retiniana anômala que é uma forma de interação binocular para a reorganização da projeção espacial ${ }^{(8)}$.

O trabalho de Hubel e Wiesel mostrou que não há amblíope adulto que possa recuperar a visão ${ }^{(9)}$, por isso, a ambliopia deve ser diagnosticada o mais precoce possível. Somente a intervenção apropriada e em tempo correto pode prevenir e reverter à perda visual por fatores ambliogênicos.

A Estratégia de Saúde da Família tem como objetivo geral contribuir para a reorientação do modelo assistencial , com os princípios do Sistema Único de Saúde, a partir da atenção básica, imprimido uma nova dinâmica de atuação, com definição de responsabilidades entre os serviços de saúde e a população ${ }^{(8)}$, com o objetivo da modificação dos paradigmas da prática das ações de saúde, através de uma ação direta coletiva dentro do ambiente físico e social da família ${ }^{(10)}$. No documento original do Ministério da Saúde, o Programa de Saúde da Família consiste em uma estratégia que prioriza as ações de promoção, proteção e recuperação da saúde da família e dos indivíduos, dos recém-nascidos, dos idosos sadios ou doentes de forma integral e contínua ${ }^{(10)}$.

\section{Métodos}

Realizado estudo tranversal para determinação da prevalência de ambliopia e de sensibilidade e especificidade para detecção da ambliopia através da medida da acuidade visual com a tabela LEA e ETDRS.

Para determinar a sensibilidade e especificidade da tabela LEA, comparamos os resultados encontrados com o padrão ouro (tabela ETDRS com exame oftalmológico completo).

\section{Unidade do PSF do estudo}

A Unidade do Programa de Saúde da Família Lapa, Rio de Janeiro está localizada dentro do Campus do Curso de Medicina da Universidade Estácio de Sá na Rua Riachuelo, Lapa, Centro, Rio de Janeiro possuindo como área adscrita, 7 microáreas

\section{Amostra do estudo}

Amostra constituída por todas as crianças de três a sete anos da região adscrita . O número total foi de 93 (listagem oferecida na própria unidade) no período de abril a setembro de 2008.

Foi realizado um primeiro encontro com as agentes comunitárias de saúde quando foram entregues convites impressos para as crianças.

Várias atividades coletivas lúdicas foram desenvolvidas para sensibilizar a população sobre a visão, tais como: pintura de olhos em bandeirinhas para a festa junina do PSF, colagem utilizando revistas especializadas em óptica e reflexões sobre o Manual da Boa Visão (elaborado e distribuído pelo Conselho Brasileiro de Oftalmologia). Foi montado um mural no dia do encerramento com o trabalho coletivo.

Foi realizado treinamento com acadêmicos do curso de medicina da UNESA que auxiliaram na pesquisa. O treinamento teórico e prático abrangeu acuidade visual, medidas de acuidade visual, erros de refração e tabelas. A ficha de exame foi apresentada aos alunos. Os alunos fizeram treinamento em anamnese e medida da visão com oftalmologista preceptora e entre eles. O padrão 
Tabela 1

Distribuição das Ametropias por olho das crianças examinadas

\begin{tabular}{lccc}
\hline Ametropia & $\begin{array}{c}\text { Frequência } \\
\text { absoluta }\end{array}$ & $\begin{array}{c}\text { Frequência } \\
\text { relativa (\%) }\end{array}$ & Prevalência \\
\hline Hipermetropia & 64 & 87,7 & 54,2 \\
Miopia & 04 & 5,5 & 3,4 \\
Astigmatismo Miópico Simples & 02 & 2,6 & 1,6 \\
Astigmatismo Hipermetrópico Simples & 01 & 1,4 & 0,85 \\
Astigmatismo Hipermetrópico Composto & 01 & 1,4 & 0,85 \\
Astigmatismo Miópico Composto & 00 & 00 & 00 \\
Astigmatismo Misto & 01 & 1,4 & 0,85 \\
Total & 73 & $100 \%$ & 61,8 \\
\hline
\end{tabular}

utilizado na medida foi a fração para ambas as tabelas.

O processo de coleta de informações foi registrado por anamnese em fichas de exame pelos alunos, com tabela para medida de acuidade visual e exame oftalmológico com termo de consentimento informado.

\section{Exame ocular}

Todas as crianças foram submetidas à avaliação oftalmológica completa que incluiu: anamnese dirigida, ectoscopia, medida da acuidade visual com tabela ETDRS e com a rtabela LEA, reflexo vermelho com oftalmoscópio direto, Teste de Titmus, refração objetiva sob cicloplegia, refração subjetiva, avaliação da motilidade ocular, biomicroscopia do segmento anterior, fundoscopia sob midríase.

$\mathrm{O}$ exame oftalmológico completo foi realizado pela mestranda e autora deste trabalho com tabela ETDRS

Os exames de acuidade visual foram feitos de forma duplo cega, com a tabela LEA testada pelos alunos.

Os critérios diagnósticos para as ambliopia e fatores ambliogênicos foram:

- Ametropia $=>$ erro refracional igual ou maior que $0,5 \mathrm{D}$ por olho.

- Ambliopia => Constituída por formas anisométricas, estrábicas, refracionais e de deprivação. As formas refracionais foram definidas como visão menor que 0,7 (20/32) na medida decimal ou maior que 0,2 na medida LogMar, sendo a refração igual ou maior que $+2,0$ esférico, $-1,00$ esférico ou -1,00 cilindro, no melhor olho, sob cilcloplegia a $75 \mathrm{~cm}$, descontando 1,5 D da distância e 1,0 D da cicloplegia.

- Estrabismo => alteração manifesta do alinhamento ocular.

- Anisometropia => diferença mínima de uma dioptria de grau entre os dois olhos ${ }^{(11)}$.
Tabela 2

Distribuição dos fatores ambliogênicos por criança

\begin{tabular}{lcc}
\hline $\begin{array}{l}\text { Fatores } \\
\text { ambliogênicos }\end{array}$ & $\begin{array}{c}\text { Frequência } \\
\text { absoluta }\end{array}$ & Prevalência \\
\hline Estrabismo & 05 & 8,4 \\
Anisometropia & 07 & 11,86 \\
Ametropia* & 9 & 15,2 \\
Ex-anopsia & 0 & 0 \\
\hline
\end{tabular}

\section{Resultados}

Foram examinadas 59 crianças (118 olhos ) que apresentaram ametropia em 73 olhos. A prevalência das ametropias na amostra estudada foi de 61,8 \% (Tabela 1).

Das crianças estudadas, 06 apresentaram ambliopia. A prevalência de ambliopia na amostra foi de $10 \%$.

Das crianças que apresentaram ambliopia 05 possuíam como fator ambliogênico o estrabismo (esotropia) e a hipermetropia (sob cicloplegia) entre $+1,00$ e $+5,00$ dioptrias.

Três crianças também apresentaram anisometropia e uma possuía miopia de - 2,50 dioptrias.

A tabela LEA para triagem de ambliopia apresentou $100 \%$ de sensibilidade e especificidade de $30,9 \%$. A sensibilidade e especificidade da tabela ETDRS foi respectivamente, $100 \%$ e $18 \%$.

\section{DiscuSsão}

Hipermetropia de $+2,75$ dioptrias associado à esotropia é significativo para a presença de ambliopia conforme a literatura médica ${ }^{(11)}$.

Anisometropia com esotropia associada à 
hipermetropia de duas ou mais dioptrias no olho mais emetrópico; diferenças de dioptrias de $+1,00$ esféricas ou -1,00 dioptrias cilíndricas são associadas à presença de ambliopia também. A presença adicional de esotropia aumenta a profundidade da ambliopia ${ }^{(11)}$. O nível de significância da associação de erros refrativos com estrabismo e ambliopia foi predominantemente mais alta com história familiar positiva para estrabismo. $\mathrm{O}$ autor encontra uma associação próxima entre o erro de refração e a presença de estrabismo e / ou ambliopia.

Ingran et al. consideram a refração como a base do rastreamento de crianças pré-escolares para a deteccção de defeitos visuais ${ }^{(11)}$.

Abelardo de Souza et al. em estudo com escolares de favelas do Alto da Boa Vista, Rio de Janeiro, Brasil, no ano de 2001 encontrou prevalência dos erros refrativos de 3,5\% (ametropias positivas, 1,78\%; ametropias negativas, $1,06 \%$ e astigmatismos mistos, $0,67 \%)$. A prevalência das oftalmopatias foi de $4,83 \%$. Ambliopia teve prevalência de 2,00\%, manifestações do estrabismo, $1,72 \%$ e outras causas $1,11 \%{ }^{(12)}$.

Diferenças do nosso trabalho podem ser expostas: foram avaliadas 1800 crianças no total. $306(17,00$ $\%$ do total ) foram encaminhadas ao Instituto Benjamin Constant. Destas, houve 183 (10,17\% do total e 59,80\% das triadas) que receberam alta por apresentarem visão melhor que 0,8 ao exame realizado durante campanha de saúde ocular na rede pública de ensino.

No nosso trabalho, o total de crianças em idade pré-escolar apresentada pela listagem do PSF foi de 93. Deste total, apenas 59 compareceram e apenas duas frequentavam a pré-escola. Trinta e quatro crianças não compareceram mesmo após diversas chamadas. Nossa prevalência de ametropias foi de $15,2 \%$ e, de estrabismo $8,4 \%$. Talvez esse fato se deva ao reduzido tamanho da amostra.

Em Sorocaba, a prevalência de baixa acuidade visual em escolares da rede pública foi de $13,1 \%{ }^{(13)}$. Essa prevalência ainda é quase o dobro da menor prevalência encontrada, 7,6\% ${ }^{(14)}$.

O trabalho, realizado no Programa de Saúde da Família na Lapa no Rio de Janeiro, demonstrou achados oculares encontrados no exame oftalmológico completo em relação às crianças triadas de $33,3 \%$ de ametropias, $8,35 \%$ de estrabismo e normalidade de $58,3 \%{ }^{(15)}$.

A comparação com o trabalho anterior publicado por Portes em 2007 demonstrou queda da prevalência das ametropias de $33,3 \%$ para $11,86 \%$ e a prevalência de estrabismo manteve -se na mesma proporção. Tal fato pode talvez ser explicado pela ausência de campanhas de saúde oculares e os diversos "nós" encontrados no sistema de referência e contrarreferência no SUS , com ausência de critérios para medida de visão pelo PSF .

Ambas tabelas ETDRS e LEA podem ser usadas para a triagem de ambliopia porque tiveram 100\% de sensibilidade. A especificidade foi maior para a tabela LEA. Porém, ainda em nível baixo. Portanto, há necessidade da complementação com o exame oftalmológico completo para confirmar e ratificar a presença de fatores ambliogênicos, incluindo as ametropias em crianças.

\section{Abstract}

Objective: To determine amblyogenic factors and amblyopia prevalence in a Health Family assisted community área of Lapa $(R J)$ and to estimate in the same area the sensiblity and specificity among visual acuity methods (ETDRS versus LEA optotypes) in pre-school children.Methods: Cross section study of 93 children from three to six years old. All the children were submitted to complete ophthalmological evaluation which included: anamnesis, ectoscopy, double blind visual acuity measurements with LEA and ETDRS optotypes, red reflex, Titmus Test, objective cycloplegic refraction, subjective refraction, ocular motility evaluation, anterior segment biomicroscopy, fundoscopy. The data were collected in Ronaldo Gazolla Policlinic of Estacio de Sá University.Results: The amblyogenic factors prevalence were: $8,4 \%$ of strabismus, $11,86 \%$ of anisometropia and $15,2 \%$ of ametropia. The ETDRS visual acuity test had $100 \%$ of sensibility and $18 \%$ of specificity for amblyopia detection. Lea visual acuity test had $100 \%$ sensibility and 30,9\% specificity for amblyopia detection.Conclusion: Both optotype tests ETDRS and LEA can be used for amblyopia screening because they had 100\% sensibility. The specificity was higher for LEA optotypes. However, the specificity still remained in a low level. Therefore, it is necessary to complement the visual acuity screening with a complete ophthalmological exam in the individuals screened to have amblyopia. Visual screening performed by Health Community Agents could identify children with visual acuity problems, and they could be referred to an ophthalmological complete exam, in an age group that visual acuity problems resolution is a priority. Helth Family Program can be used to evaluate visual acuity children status and it would be useful that this evaluation could be a statiscal SUS mark.

Keywords: Amblyopia/diagnosis; Prevalence; Visual acuity; Family health program; Child; Child, preschool; Period analysis; Sensitivity and Specificity 


\section{REFERÊNCIAS}

1. Alberto FL, Callera F, Daré GN, Rodrigues ML. Estudo das condições oculares em uma população de estudantes de primeiro grau na cidade de Ribeirão Preto. Arq Bras Oftalmol. 1992; 5:188.

2. Yamane Y. Estrabismo. In: Dantas AM. Oftamologia pediátrica. Rio de Janeiro: Cultura Médica; 1995. p. 455.

3. Slamovits T. Basic and clinical science course: Section 8, 19961997. San Francisco: American Academy of Ophthalmology; 1996. p.275-8.

4. Consoni Filho E. Fisiopatologia da ambliopia. An Oftalmol. 1988; 7(1): 22-23.

5. Romani FA. Estudo oftalmológico em escolares da cidade de Jaraguá do Sul (SC). Arq Bras Oftalmol. 1981;44(4):143-4.

6. Lauretti Filho A, Romão E.. Estudo da acuidade visual e dos erros de refração em crianças com baixo rendimento escolar. Rev Bras Oftalmol. 1982; 41(5):31-6.

7. Bicas HE. Fisiologia da visão binocular. Arq Bras Oftalmol. 2004; 67(1):172-80.

8. Brasil. Ministério da Saúde. Secretaria de Assistência à Saúde. Coordenação de Saúde da Comunidade. Saúde da família: uma estratégia para a reorientação do modelo assistencial. Brasília. Ministério da Saúde; 1997.
9. Brik M.. Prevenção da ambliopia. An Oftalmol. 1988; 7 (1):57-60.

10. Brasil. Ministério da Saúde. Programa de saúde da família [Internet]. Brasília: Ministério da Saúde; 1994 [citado 2010 Fev 2]. Disponível em: <http://portal.saude.gov.br/portal/ aplicacoes/noticias>

11. Ingram RM. Refraction as a basis for screening children for squint and amblyopia. Br J Ophthalmol. 1977;61(1):8-15.

12. Couto Júnior AS, Pinto GR, Oliveira DA, Holzmeister D, Portes AL, Neurauter R, et al. Prevalência das ametropias e oftalmopatias em crianças pré-escolares e escolares em favelas do Alto da Boa Vista, Rio de Janeiro, Brasil. Rev Bras Oftalmol. 2007; 66(5):304-8.

13. Gianini RJ, Masi E, Coelho EC, Oréfice FR, Moraes RA. Prevalência de baixa acuidade visual em escolares da rede pública, Sorocaba. Rev Saúde Pública. 2004; 38(2):201-8.

14. Temporini ER. Aspectos do plano de oftalmologia sanitária escolar do estado de São Paulo. Rev Saúde Pública. 1982;16(4):243-60.

15. Portes AJ, Portes AL, Bonfadini CG, Shinzato FA, Pecego MG. Prevenção à cegueira em crianças de três a seis anos de idade assistidas pelo Programa de Saúde da Família - Lapa, Rio de Janeiro. Rev Bras Oftalmol. 2007; 66 (3):155-9. 